\title{
Classical Lie subalgebras of the Lie algebra of matrix differential operators on the circle
}

\author{
Carina Boyallian ${ }^{\mathrm{a})}$ and Jose I. Liberati ${ }^{\mathrm{b})}$ \\ CIEM-FAMAF Universidad Nacional de Córdoba, 500 Córdoba, Argentina
}

(Received 15 November 2000; accepted for publication 28 March 2001)

We give a complete description of the anti-involutions of the algebra $\mathcal{D}^{N}$ of $N$ $\times N$-matrix differential operators on the circle, preserving the principal $\mathbb{Z}$ gradation. We obtain, up to conjugation, two families $\sigma_{ \pm, m}$ with $1 \leqslant m \leqslant N$, getting two families $\mathcal{D}_{ \pm, m}^{N}$ of simple Lie subalgebras fixed by $-\sigma_{ \pm, m}$. We also give a geometric realization of $\sigma_{ \pm, m}$, concluding that $\mathcal{D}_{+, m}^{N}$ is a subalgebra of $\mathcal{D}^{N}$ of type $o(m, n)$ and $\mathcal{D}_{-, m}^{N}$ is a subalgebra of $\mathcal{D}^{N}$ of type $\operatorname{osp}(m, n)$ (ortho-symplectic). Finally, we study the conformal algebras associated with $\mathcal{D}_{+, m}^{N}$ and $\mathcal{D}_{-, m}^{N}$. $@ 2001$ American Institute of Physics. [DOI: 10.1063/1.1380252]

\section{INTRODUCTION}

Certain subalgebras of the Lie algebra $\mathcal{D}$ of differential operators on the circle were considered in Ref. 1, where they showed that there are two, up to conjugation, anti-involution $\sigma_{ \pm}$on $\mathcal{D}$, which preserve the principal gradation, and they obtained in this way two different Lie subalgebras fixed by $-\sigma_{ \pm}$. The main goal of the present paper is to extend the results in Ref. 1 to the matrix case, where the picture seems to be rather more complicated and richer.

We give a complete description of the anti-involutions of the algebra $\mathcal{D}^{N}$ (whose central extension is usually denoted by $\left.W_{1+\infty}^{N}\right)$, of $N \times N$-matrix differential operators on the circle, preserving the principal $\mathbb{Z}$ gradation. We obtain, up to conjugation, two families $\sigma_{ \pm, m}$ with 1 $\leqslant m \leqslant N$, getting two families $\mathcal{D}_{ \pm, m}^{N}$ of Lie subalgebras fixed by $-\sigma_{ \pm, m}$, and we show that these subalgebras are simple. Then, we give a geometric realization of $\sigma_{ \pm, m}$, concluding that $\mathcal{D}_{+, m}^{N}$ is a subalgebra of $\mathcal{D}^{N}$ of type $o(m, n)$ and $\mathcal{D}_{-, m}^{N}$ is a subalgebra of $\mathcal{D}^{N}$ of type $\operatorname{ssp}(m, n)$ (orthosymplectic).

Finally, we study the conformal algebra associated with $\mathcal{D}_{+, m}^{N}$. Following the notation in Ref. 2 , recall that $\mathcal{D}^{N}$ is a formal distribution algebra with the family of pairwise local formal distributions $\mathcal{F}=\left\{J_{A}^{n}(z)=\delta(t-z)\left(-\partial_{t}\right)^{n} \otimes A: n \in \mathbb{Z}_{+}, A \in \mathrm{Mat}_{N} \mathrm{C}\right\}$ (see Ref. 2, Example 2.10), and the associated associative (respectively, Lie) conformal algebra is $\operatorname{Cend}_{N}=\overline{\mathcal{F}}$ (respectively, $g c_{N}$ ). When we try to extend the anti-involutions $\sigma_{+, m}$ on $\mathcal{D}^{N}$ to the associative conformal algebra $\operatorname{Cend}_{N}$ we find some problems. If we apply $\sigma_{+, m}$ to the fields $J_{A}^{k}$, we have that $\sigma_{+, m}(\mathcal{F}) \oplus \overline{\mathcal{F}}$, except in the degenerated case $m=0$. Using the notion of $\Gamma$-twisted and $\Gamma$-formal distribution algebras ( $\Gamma$ is a group) introduced in Refs. 3 and 4, we are able to characterize the conformal subalgebras of $g c_{N}$ associated with $\mathcal{D}_{+, m}^{N}$ and $\mathcal{D}_{-, m}^{N}$. In the case of $\mathcal{D}_{+, m}^{N}$, we get the conformal orthogonal subalgebra $o c_{N}$ of $g c_{N}$ with a $\mathbb{Z}_{2}$-gradation, and in the other case, $\mathcal{D}_{-, m}^{N}$, the associated conformal algebra is $g c_{N}$ with a $\mathbb{Z}_{2} \times \mathbb{Z}_{2}$ action given by a $\mathbb{Z}_{2}$-gradation and an action of $\mathbb{Z}_{2}$ (multiplicative) by semilinear automorphisms.

In Ref. 5, the representation theory of the central extension of $\mathcal{D}^{N}\left(W_{1+\infty}^{N}\right)$ was studied and it may be interesting to develop the representation theory for this new family of simple Lie subalgebras (see Ref. 1 for the special case $N=1$ ).

The paper is organized as follows. In Sec. II we review the Lie algebra $\mathcal{D}^{N}$ and classify the anti-involutions of $\mathcal{D}^{N}$ preserving the principal Z-gradation of $\mathcal{D}^{N}$. In Sec. III, we describe the Lie

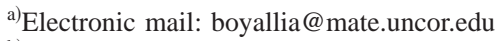

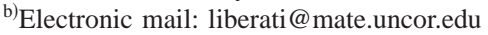


subalgebras $\mathcal{D}_{ \pm, \mathrm{m}}^{\mathcal{N}}$. In Sec. IV we prove that $\mathcal{D}_{ \pm, \mathrm{m}}^{\mathcal{N}}$ are simple. In Sec. V, we obtain a geometric realization of $\sigma_{+, m}$. In Sec. VI we study the conformal subalgebra associated with $\mathcal{D}_{+\mathrm{m}}^{\mathcal{N}}$, and in Sec. VII, the conformal subalgebra associated with $\mathcal{D}_{-, \mathrm{m}}^{\mathcal{N}}$.

We are very grateful to Victor Kac for useful discussions and suggestions about this paper, and for introducing us to this subject, particularly what we have learned about the symbol map and the identification $g c_{n}=\operatorname{Mat} \mathrm{C}[\partial, x]$ from his lectures at MIT in Spring'98, used in Sec. VI and VII.

\section{ANTI-INVOLUTIONS OF $\mathcal{D}^{N}$}

Let $N$ be a positive integer. Denote by $\mathcal{D}_{\text {as }}^{N}$ the associative algebra of all regular matrix differential operators on $\mathrm{C}^{\times}$, i.e., the operators on $\mathbb{C}^{N}\left[t, t^{-1}\right]$ of the form

$$
\begin{gathered}
E=e_{k}(t) \partial_{t}^{k}+e_{k-1}(t) \partial_{t}^{k-1}+\cdots+e_{0}(t), \\
\text { where } e_{i}(t) \in \operatorname{Mat}_{N} \mathrm{C}\left[t, t^{-1}\right] .
\end{gathered}
$$

Here and in the following we denote by $\operatorname{Mat}_{N} R$ the associative algebra of all $N \times N$ matrices over an algebra $R$. It is more convenient to write the differential operators as linear combinations of elements of the form $t^{k} f(D) A$, where $f$ is a polynomial, $D=t \partial_{t}, k \in \mathbb{Z}$, and $A \in \mathrm{Mat}_{N} \mathrm{C}$. The product in $\mathcal{D}_{\text {as }}^{N}$ is then given by

$$
\left(t^{r} f(D) A\right)\left(t^{s} g(D) B\right)=t^{r+s} f(D+s) g(D) A B .
$$

Let $\mathcal{D}^{N}$ denote Lie algebra obtained from $\mathcal{D}_{\text {as }}^{N}$. The bracket in $\mathcal{D}^{N}$ may be conveniently calculated by the following formula:

$$
\left[t^{r} f(D) A, t^{s} g(D) B\right]=t^{r+s}(f(D+s) g(D) A B-f(D) g(D+r) B A) .
$$

The elements $t^{k} D^{m} E_{i j}\left(k \in \mathbb{Z}, m \in \mathbb{Z}_{+}, i, j=1, \ldots, N\right)$ form a basis of $\mathcal{D}^{N}$. Here and in the following $E_{i j}$ is the standard basis of $\mathrm{Mat}_{N} \mathrm{C}$. Define the weight wt on $\mathcal{D}^{N}$ by

$$
\text { wt } t^{k} f(D) E_{i j}=k N+i-j .
$$

This gives us the principal Z-gradation of $\mathcal{D}_{\text {as }}^{N}$ and $\mathcal{D}^{N}: \mathcal{D}^{N}=\oplus_{j \in \mathbb{Z}} \mathcal{D}_{j}^{N}$, and so we have the triangular decomposition

$$
\mathcal{D}^{N}=\mathcal{D}^{N}+\oplus \mathcal{D}^{N}{ }_{0} \oplus \mathcal{D}^{N},
$$

where $\mathcal{D}^{N}{ }_{ \pm}=\oplus_{j \in \pm N} \mathcal{D}^{N}{ }_{j}$.

An anti-involution $\sigma$ of $\mathcal{D}_{\mathrm{as}}^{N}$ is an involutive antiautomorphism of $\mathcal{D}_{\mathrm{as}}^{N}$, i.e., $\sigma^{2}=I, \sigma(a X$ $+b Y)=a \sigma(X)+b \sigma(Y)$ and $\sigma(X Y)=\sigma(Y) \sigma(X)$, for all $a, b \in \mathrm{C}, X, Y \in \mathcal{D}_{\mathrm{as}}^{N}$.

In order to classify the anti-involutions of $\mathcal{D}_{\text {as }}^{N}$ preserving its principal gradation, we shall need the following notation. Define for each $m=1, \ldots, N$, the permutation $\pi_{m}$ in $S_{N}$ given by

$$
\begin{array}{ccccccccc}
1 & 2 & \ldots & m-1 & m & m+1 & \ldots & N-1 & N \\
\downarrow & \downarrow & & \downarrow & \downarrow & \downarrow & & \downarrow & \downarrow \\
m & m-1 & \ldots & 2 & 1 & N & \ldots & m+2 & m+1
\end{array} .
$$

Let us fix $m=1, \ldots, N, f \in \mathrm{C}$ and $c=\left\{c_{i, j}\right\}, c_{i, j} \in \mathrm{C}, i>j$, we define $\sigma=\sigma_{ \pm, f, c, m}$ by the following formulas:

$$
\begin{gathered}
\sigma\left(E_{i i}\right)=E_{\pi_{m}(i), \pi_{m}(i)}, \\
\sigma\left(D E_{i i}\right)=\left(-D+f-\delta_{i>m}\right) E_{\pi_{n}(i), \pi_{m}(i)},
\end{gathered}
$$




$$
\begin{aligned}
& \sigma\left(t E_{i i}\right)= \pm t E_{\pi_{m}(i), \pi_{m}(i)}, \\
& (i>j) \quad \sigma\left(E_{i, j}\right)=\left\{\begin{array}{cc}
c_{i, j} E_{\pi_{m}(j), \pi_{m}(i)} & \text { if } \pi_{m}(i) \leqslant N-i+j \\
t c_{i, j} E_{\pi_{m}(j), \pi_{m}(i)} & \text { if } N-i+j<\pi_{m}(i)
\end{array},\right. \\
& (i<j) \quad \sigma\left(E_{i, j}\right)=\left\{\begin{array}{lll}
t^{-1} c_{j, i}^{-1} E_{\pi_{m}(j), \pi_{m}(i)} & \text { if } \quad \pi_{m}(i) \leqslant j-i \\
c_{j, i}^{-1} E_{\pi_{m}(j), \pi_{m}(i)} & \text { if } & \pi_{m}(i)>j-i
\end{array},\right.
\end{aligned}
$$

where

$$
\delta_{i>m}= \begin{cases}1 & \text { if } i>m \\ 0 & \text { if } i \leqslant m\end{cases}
$$

Theorem 1: Let $m=1, \ldots, N, f \in \mathrm{C}$, and $c=\left\{c_{i, j}\right\}, c_{i, j} \in \mathrm{C}, i>j$. Then $\sigma=\sigma_{ \pm, f, c, m}$ defined on generators by (2.6) extends to an anti-involution on $\mathcal{D}_{\text {as }}^{N}$ which preserves the principal Z-gradation if and only if

$$
c_{i, j}=c_{i, i-1} c_{i-1, i-2} \ldots c_{j+1, j}
$$

and

$$
\left\{\begin{array}{l}
c_{i, j} c_{\pi_{m}(j), \pi_{m}(i)}=1 \quad \text { if } \quad \pi_{m}(i) \leqslant N-i+j, \\
c_{i, j} c_{\pi_{m}}^{-1}(i), \pi_{m}(j)= \pm 1 \quad \text { if } \quad \pi_{m}(i)>N-i+j .
\end{array} .\right.
$$

Moreover, any anti-involution $\sigma$ of $\mathcal{D}_{\text {as }}^{N}$ which preserves the principal Z-gradation is one of them.

The proof of this Theorem is certainly more difficult than the one given in Ref. 1 for $N=1$, and it will be given in several steps. We mainly use the relations between the generators $E_{i, j}$ and the involutive property of $\sigma$.

Proof: Step 1. Since $\sigma$ should preserve the principal Z-gradation, we have $\sigma\left(E_{i, i}\right)$ $=\sum_{j=1}^{N} Q_{i, j}(D) E_{j, j}$. But $\sigma\left(E_{i, i}\right)=\sigma\left(E_{i, i} E_{i, i}\right)=\sum_{j=1}^{N}\left(Q_{i, j}(D)\right)^{2} E_{j, j}$. Therefore, if $Q_{i, j}(D) \neq 0$, then $Q_{i, j}(D) \equiv 1$. Note that $Q_{i, j}$ is independent of $D$. Now, $E_{i, i}=\sigma^{2}\left(E_{i, i}\right)=\sum_{k, j=1}^{N} Q_{i, j} Q_{j, k} E_{k, k}$, then $\delta_{i, k}=\Sigma_{j=1}^{N} Q_{i, j} Q_{j, k}$. So, for each $i$ there exist a unique $j_{i}$ such that $Q_{i, j_{i}} Q_{j_{i, i}}=1$, then $Q_{j_{i}, i}$ $=1$. On the other hand, we also have $Q_{j_{i},} Q_{i, k}=0$ for any $k \neq j_{i}$, then $Q_{i, k}=0$ for any $k \neq j_{i}$, obtaining that $\sigma\left(E_{i, i}\right)=E_{j_{i}, j_{i}}$. Due to the injectivity of $\sigma, \pi(i):=j_{i}$ is a permutation in $S_{N}$, and since $\sigma$ is an involution, we have $\pi^{2}=i d$.

Similar ideas will be used in the following steps.

Step 2. Again, using that $\sigma$ should preserve the principal Z-gradation, we may assume that $\sigma\left(D E_{i, i}\right)=\sum_{j=1}^{N} P_{i, j}(D) E_{j, j}$. We have

$$
\begin{aligned}
\sigma\left(D E_{i, i}\right)=\sigma\left(D E_{i, i} E_{i, i}\right) & =\sigma\left(E_{i, i}\right) \sigma\left(D E_{i, i}\right) \\
& =E_{\pi(i), \pi(i)}\left(\sum_{j=1}^{N} P_{i, j}(D) E_{i, j}\right) \\
& =P_{i, \pi(i)}(D) E_{\pi(i), \pi(i)} .
\end{aligned}
$$

Therefore, $\sigma\left(D E_{i, i}\right)=P_{i}(D) E_{\pi(i), \pi(i)}$ with $P_{i}(D)=P_{i, \pi(i)}(D)$. Now, let us write $P_{i}(D)$ $=\bar{P}_{i}(D)+\dot{P}_{i}$, where $\dot{P}_{i}$ stands for the constant term of $P_{i}(D)$. Thus, 


$$
\begin{aligned}
D E_{i, i} & =\sigma^{2}\left(D E_{i, i}\right)=\sigma\left(P_{i}(D) E_{\pi(i), \pi(i)}\right) \\
& =\sigma\left(\left(\bar{P}_{i}(D)+\dot{P}_{i}\right) E_{\pi(i), \pi(i)}\right) \\
& =\bar{P}_{i}\left(\sigma\left(D E_{\pi(i), \pi(i)}\right)\right)+\dot{P}_{i} \sigma\left(E_{\pi(i), \pi(i)}\right) \\
& =\left(\bar{P}_{i}\left(P_{\pi(i)}(D)\right)+\dot{P}_{i}\right) E_{i, i} .
\end{aligned}
$$

So, we have $D=\bar{P}_{i}\left(P_{\pi(i)}(D)\right)+\dot{P}_{i}$. Thus, $P_{i}(D)=a_{i} D+f_{i}$ with $a_{i} \cdot a_{\pi(i)}=1$ and $a_{i} \cdot f_{\pi(i)}+f_{i}$ $=0$.

Step 3. Let us suppose that $\sigma\left(t E_{i, i}\right)=t \Sigma_{l=1}^{N} T_{i, l}(D) E_{l, l}$. Using a similar argument to the one used in (2.8), we can deduce that $T_{i, k}(D) \equiv 0$ if $k \neq \pi(i)$. As before, we denote $T_{i}(D)=T_{i, \pi(i)}$ $\times(D)$. Again, let us write $T_{i}(D)=\bar{T}_{i}(D)+\dot{T}_{i}$, where $\dot{T}_{i}$ stands for the constant term of $T_{i}(D)$. Thus,

$$
\begin{aligned}
t E_{i, i}=\sigma^{2}\left(t E_{i, i}\right) & =\sigma\left(t T_{i}(D) E_{\pi(i), \pi(i)}\right) \\
& =\sigma\left(\left(t E_{\pi(i), \pi(i)}\right)\left(\bar{T}_{i}(D)+\dot{T}_{i}\right) E_{\pi(i), \pi(i)}\right) \\
& =\left[\bar{T}_{i}\left(\sigma\left(D E_{\pi(i), \pi(i)}\right)+\dot{T}_{i} \sigma E_{\pi(i), \pi(i)}\right] \sigma\left(t E_{\pi(i), \pi(i)}\right)\right. \\
& =\left(\bar{T}_{i}\left(T_{\pi(i)}\left(a_{\pi(i)} D+f_{\pi(i)}\right)\right)+\dot{T}_{i}\right) t T_{\pi(i)}(D) E_{i, i} \\
& =t\left(\left(\bar{T}_{i}\left(T_{\pi(i)}\left(a_{\pi(i)}(D+1)+f_{\pi(i)}\right)\right)+\dot{T}_{i}\right) T_{\pi(i)}(D)\right) E_{i, i}
\end{aligned}
$$

Thus, $1=T_{\pi(i)}(D) \quad\left(\bar{T}_{i}\left(T_{\pi(i)}\left(a_{\pi(i)}(D+1)+f_{\pi(i)}\right)\right)+\dot{T}_{i}\right)$. Then $\operatorname{deg}\left(T_{i}\right)=0$ for all $i$, and $T_{i}$ $T_{\pi(i)}=1$.

Step 4. Suppose $i>j$. Considering the Z-gradation, we have that $\sigma\left(E_{i, j}\right)$ $=\Sigma_{l=1}^{N-i+j} C_{l}^{i j}(D) E_{l+i-j, l}+\sum_{l=N-i+j+1}^{N} t C_{l}^{i j}(D) E_{l+i-j-N, l}$. Since, $\sigma\left(E_{l, l} E_{i, j}\right)=\sigma\left(E_{i, j}\right) \sigma\left(E_{l, l}\right)$ $=\sigma\left(E_{i, j}\right) E_{\pi(l), \pi(l)}$, we can deduce $C_{\pi(l)}^{i, j}(D)=0$ for all $l \neq i$. Let $C^{i, j}(D)=C_{\pi(i)}^{i, j}(D)$. Therefore, we have

$$
\sigma\left(E_{i, j}\right)= \begin{cases}C^{i, j}(D) E_{\pi(i)+i-j, \pi(i)} & \text { if } \\ t C^{i, j}(D) E_{\pi(i)+i-j-N, \pi(i)} & \text { if } \quad \pi(i) \geqslant N-i+j+1 .\end{cases}
$$

Similarly, if $i<j$ and

$$
\sigma\left(E_{i, j}\right)=\sum_{l=1}^{j-i} t^{-1} S_{l}^{i j}(D) E_{N+l+i-j, l}+\sum_{l=j-i+1}^{N} S_{l}^{i j}(D) E_{l+i-j, l},
$$

we deduce that $S_{\pi(l)}^{i, j}(D)=0$ for all $l \neq i$. Thus, denoting $S^{i, j}(D):=S_{\pi(i)}^{i j}(D)$, we have

$$
\sigma\left(E_{i, j}\right)=\left\{\begin{array}{l}
S^{i, j}(D) E_{\pi(i)+i-j, \pi(i)} \quad \text { if } \quad \pi(i) \geqslant j-i+1 \\
t^{-1} S^{i, j}(D) E_{\pi(i)+i-j+N, \pi(i)} \quad \text { if } \pi(i) \leqslant j-i .
\end{array}\right.
$$

Let $i>j$ and $k=i-j$, since $\sigma$ is an involution we have if $\pi(i) \leqslant N-i+j$ :

$$
E_{i, j}=\sigma^{2}\left(E_{i, j}\right)=\sigma\left(C^{i, j}(D) E_{\pi(i)+k, \pi(i)}\right)=\sigma\left(C^{i, j}(D) E_{\pi(i)+k, \pi(i)+k} E_{\pi(i)+k, \pi(i)}\right)
$$

using (2.9), we must have $\pi(\pi(i)+k)+k \leqslant N-k$, otherwise we get $t$ in the right-hand side above, so $E_{i, j}=C^{\pi(i)+k, \pi(i)}(D) C^{i, j}\left(a_{\pi(i)+k} D+f_{\pi(i)+k}\right) E_{\pi(\pi(i)+k)+k, \pi(\pi(i)+k)}$. Then, $c_{i, j}:=C^{i, j}(D)$ are constant, $j=\pi(\pi(i)+k)$ and $c_{i, j} \cdot c_{\pi(j), \pi(i)}=1$, using $\pi^{2}=i d$. 
If $\pi(i) \geqslant N-i+j+1$, in the same way, using simultaneously (2.9) and (2.10) and taking care of the $t$ that appears in $\sigma\left(E_{i, j}\right)$ we have $d_{\pi(j), \pi(i)}:=S^{\pi(j), \pi(i)}(D)$ are constant, $j=\pi(\pi(i)+k$ $-N)$, and $1=c_{i, j} \cdot d_{\pi(j), \pi(i)} \cdot b_{\pi(j)}$.

Consider $i<j$ and take $k=i-j$, by the same argument, if $\pi(i) \geqslant-k+1$ then we have $d_{i, j}$ $:=S^{i, j}(D)$ are constant, $j=\pi(\pi(i)+k)$, and $d_{i, j} \cdot d_{\pi(j), \pi(i)}=1$.

And, if $\pi(i) \leqslant-k$ then we have $j=\pi(\pi(i)+N+k)$ and $d_{i, j} \cdot c_{\pi(j), \pi(i)} \cdot b_{\pi(j)}^{-1}=1$.

Step 5. Let $i>j$, then by step $1, E_{\pi(i), \pi(i)}=\sigma\left(E_{i, i}\right)=\sigma\left(E_{i, j} \cdot E_{j, i}\right)=\sigma\left(E_{j, i}\right) \cdot \sigma\left(E_{i, j}\right)$, and using (2.9) and (2.10) we get

$$
d_{j, i} \cdot c_{i, j}=1 .
$$

Now, let us determine the permutation $\pi$. Again, we have for $i=2, \ldots, N, E_{\pi(i), \pi(i)}=\sigma\left(E_{i, i}\right)$ $=\sigma\left(E_{i, i-1} \cdot E_{i-1, i}\right)=\sigma\left(E_{i-1, i}\right) \cdot \sigma\left(E_{i, i-1}\right)$. Now, rewriting (2.9) and (2.10) for this case, we have

$$
\begin{aligned}
& \sigma\left(E_{i, i-1}\right)=\left\{\begin{array}{l}
c_{i, i-1} E_{\pi(i)+1, \pi(i)} \quad \text { if } \pi(i)<N \\
t c_{i, i-1} E_{1, N} \quad \text { if } \pi(i)=N,
\end{array}\right. \\
& \sigma\left(E_{i-1, i}\right)=\left\{\begin{array}{l}
d_{i-1, i} E_{\pi(i-1)-1, \pi(i-1)} \quad \text { if } \pi(i-1)>1 \\
t^{-1} d_{i-1, i} E_{N, 1} \quad \text { if } \pi(i-1)=1 .
\end{array}\right.
\end{aligned}
$$

Let $i_{0}$ be such that $\pi\left(i_{0}\right)=N$. From these equations, it is easy to see that

$$
\pi\left(i_{0}-1\right)=1 \text {, and } \pi(i-1)=\pi(i)+1 \text { for any } i \neq i_{0} .
$$

Since $\pi$ is a bijective map, we conclude that $\pi$ must be $\pi_{m}$ given in (2.5) where $m=i_{0}-1$.

Step 6. In this step we will characterize the constants $a_{i}, f_{i}$, and $b_{i}$ defined in steps 2 and 3.

Let us start with $a_{i}$. We have

$$
\begin{aligned}
-b_{i} t E_{\pi(i), \pi(i)} & =-\sigma\left(E_{i, i}\right) \\
& =\left[\sigma\left(D E_{i, i}\right), \sigma\left(t E_{i, i}\right)\right] \\
& =\left[\left(a_{i} D+f_{i}\right) E_{\pi(i), \pi(i)}, b_{i} t E_{\pi(i), \pi(i)}\right] \\
& =b_{i} a_{i} t E_{\pi(i), \pi(i)} .
\end{aligned}
$$

So, $a_{i}=-1$ for all $i$. Since $t E_{i+1, i+1} \cdot E_{i+1, i}=E_{i+1, i} \cdot t E_{i, i}$, applying $\sigma$ to both sides and using (2.12) we deduce that $b_{i+1}=b_{i}$. In step 3 we showed that $b_{i} \cdot b_{\pi(i)}=1$, then $b_{i}= \pm 1$.

Finally, by applying the same argument to $D E_{i+1, i+1} \cdot E_{i+1, i}=E_{i+1, i} \cdot D E_{i, i}$, we get $f_{i_{0}}$ $=f_{i_{0-1}}-1$, and if $i \neq i_{0}$ then $f_{i}=f_{i-1}(1<i)$. Thus $f:=f_{1}=\cdots=f_{i_{0-1}}$ and $f-1=f_{i_{0}}=\cdots=f_{N}$, getting in this way all the conditions in our theorem and all the equations in (2.7).

On the other hand, it is straightforward to check that $\sigma$ defined by (2.6) is indeed antiinvolution of $\mathcal{D}_{\text {as }}^{N}$, finishing the proof.

Let us study conditions (2.7a) and (2.7b) in more detail. By (2.7a), all the coefficients $c_{i, j}$ are completely determined by

$$
c_{i}:=c_{i+1, i}, \quad i=1, \ldots, N-1,
$$

and condition (2.7b) is equivalent to $c_{i} \cdot c_{\pi_{m}(i+1)}=1(i \neq m)$, and $\pm 1=c_{m} \cdot\left(c_{N, 1}\right)^{-1}=c_{m}$ $\cdot \Pi_{i}\left(c_{i}\right)^{-1}=\Pi_{i \neq m}\left(c_{i}\right)^{-1}$. Observe that the permutation $\pi_{m}$ is basically given by two simple permutations of the sets $\{1, \ldots, m\}$ and $\{m+1, \ldots, N\}$. Thus Eq. (2.7b) reduces to

$$
c_{i} c_{m-i}=1 \quad(1 \leqslant i<m), \quad c_{m+i} c_{N-i}=1 \quad(1 \leqslant i<N-m), \quad \pm 1=\prod_{i \neq m} c_{i} .
$$


Let $N=n+m$. If $m$ (respectively, $n$ ) is even, we have $\Pi_{i<m} c_{i}=c_{(m-1) / 2}$ and $\left(c_{(m-1) / 2}\right)^{2}=1$ (respectively, $\Pi_{i>m} c_{i}=c_{m+(n-1) / 2}$ and $\left.\left(c_{m+(n-1) / 2}\right)^{2}=1\right)$; we shall call the coefficient $c_{(m-1) / 2}$ (respectively, $c_{m+(n-1) / 2}$ ) a fixed point. If $m$ or $n$ are odd, the corresponding products are equal to 1. Therefore, we have

Case -:

If $N$ is even and

(1) $m$ even, then there are two fixed points and one of them must be 1 and the other one equal to -1 .

(2) $m$ odd, then there are no fixed points and the last condition in (2.16) is impossible. Thus there is no anti-involution in this case.

If $N$ is odd, then $m$ or $n$ is even and we have only one fixed point that must be equal to -1 .

Case +:

For any $N$, the last condition in (2.16) will be satisfied if we take the (possible) fixed points equal to 1 .

\section{LIE SUBALGEBRAS $\mathcal{D}_{ \pm, m}^{N}$.}

Let $\mathcal{D}_{ \pm, f, c, m}^{N}$ denote the Lie subalgebra of $\mathcal{D}^{N}$ fixed by $-\sigma_{ \pm, f, c, m}$, namely

$$
\mathcal{D}_{ \pm, f, c, m}^{N}=\left\{a \in \mathcal{D}^{N} \mid \sigma_{ \pm, f, c, m}(a)=-a\right\} .
$$

Now, we shall study the relation among $\mathcal{D}_{ \pm, f, c, m}^{N}$ for different data $( \pm, f, c, m)$. Let $s \in \mathrm{C}$, denote by $\Theta_{s}$ the automorphism of $\mathcal{D}_{\text {as }}^{N}$ given by $\Theta_{s}(A)=A, \Theta_{s}(t I)=t I$ and $\Theta_{s}(D I)=(D$ $+s) I$, where $I$ stands for the identity matrix. Clearly $\Theta_{s}$ preserves the principal Z-gradation of $\mathcal{D}_{\text {as }}^{N}$. Let $\sigma_{f}:=\sigma_{ \pm, f, c, m}$, then we have

$$
\sigma_{f} \cdot \Theta_{s}=\sigma_{f+s}=\Theta_{s} \cdot \sigma_{f} .
$$

Similarly, let $r=\left\{r_{i, j}\right\}(i>j)$ satisfying (2.7a) and (2.7b). Denote by $\Gamma_{r}$ the automorphism of $\mathcal{D}_{\text {as }}^{N}$ defined by $\Gamma_{r}(t I)=t I, \Gamma_{r}(D I)=D I, \Gamma_{r}\left(E_{i, i}\right)=E_{i, i}, \Gamma_{r}\left(E_{i, j}\right)=r_{i, j} E_{i, j}(i>j)$, and $\Gamma_{r}\left(E_{i, j}\right)$ $=\left(r_{i, j}\right)^{-1} E_{i, j}(i<j)$. Let $\sigma_{c}:=\sigma_{ \pm, f, c, m}$, then we have

$$
\sigma_{c} \cdot \Gamma_{r}=\sigma_{c \cdot r}=\Gamma_{r^{-1}} \cdot \sigma_{c}
$$

where $(c \cdot r)_{i, j}:=c_{i, j} r_{i, j}$ and $\left(r^{-1}\right)_{i, j}=r_{i, j}^{-1}$. Observe that $c \cdot r$ and $r^{-1}$ also satisfy (2.7a) and (2.7b). Using (3.2) and (3.3), we have:

Lemma 1: (cf. Ref. 1, Lemma 2.2) (a) The Lie algebras $\mathcal{D}_{ \pm, f, c, m}^{N}$ are all isomorphic for different $f \in \mathrm{C}$. In fact, we have $\Theta_{s}\left(\mathcal{D}_{ \pm, f, c, m}^{N}\right)=\mathcal{D}_{ \pm, f-2 s, c, m}^{N}$.

(b) $\Gamma_{r}\left(\mathcal{D}_{ \pm, f, c, m}^{N}\right)=\mathcal{D}_{ \pm, f, c \cdot r^{-2, m}}^{N}$.

Due to Lemma 1 we may choose a Lie algebra among $\mathcal{D}_{ \pm, f, c, m}^{N}$, but we must keep in mind the analysis of the fixed points for the cases + and - that we made before.

We will fix $f=0$. In this way, we have a normalization similar to the one taken in Ref. 1.

Due to Lemma 1(b), it is possible to change $c$ by $c \cdot r^{-2}$. Thus we can take $c_{i}=1$, except for the fixed points that are 1 or -1 , and they should keep the sign. Denote by $\sigma_{ \pm, m}$ and $\mathcal{D}_{ \pm, m}^{N}$ the anti-involution $\sigma_{ \pm, f, c, m}$ and the Lie subalgebra $\mathcal{D}_{ \pm, f, c, m}^{N}$, respectively, with this choice of $f$ and $c$.

Remark 1: Observe that $\mathcal{D}_{ \pm, m}^{N}$ is naturally isomorphic to $\mathcal{D}_{ \pm, N-m}^{N}$.

In order to give an explicit description of this family of subalgebras, we need some notation. For any matrix $A \in \mathrm{Mat}_{m \times n}(\mathrm{C})$, define

$$
\left(A^{\dagger}\right)_{i, j}=A_{n+1-j, m+1-i},
$$

i.e., the transpose with respect to the other diagonal. Recall the anti-involutions on $\mathcal{D}:=\mathcal{D}^{1}$ given in Ref. 1: 


$$
\dot{\sigma}_{ \pm, b}\left(t^{k} f(D)\right)=( \pm t)^{k} f(-D-k+b) \quad(b \in \mathrm{C}) .
$$

We extend $\dot{\sigma}_{ \pm, b}$ to a map on $\operatorname{Mat}_{m \times n}(\mathcal{D})=\mathcal{D} \otimes \operatorname{Mat}_{m \times n}(\mathrm{C})$ by taking $\left[\dot{\sigma}_{ \pm, b}(A)\right]_{i, j}=\dot{\sigma}_{ \pm, b}\left(A_{i, j}\right)$. Case + :

We define the following maps on $\operatorname{Mat}_{m \times n}(\mathcal{D})$ :

$$
\begin{aligned}
& A^{\dagger 1}=\dot{\sigma}_{+, 0}\left(A^{\dagger}\right), \quad B^{\dagger_{2}}=t^{-1} \dot{\sigma}_{+, 0}\left(B^{\dagger}\right), \\
& C^{\dagger_{3}}=t \dot{\sigma}_{+,-1}\left(C^{\dagger}\right), \quad F^{\dagger_{4}}=\dot{\sigma}_{+,-1}\left(F^{\dagger}\right) .
\end{aligned}
$$

Then the anti-involution $\sigma_{+, m}$ on $\mathcal{D}^{N}=\mathcal{D} \otimes \operatorname{Mat}_{N}(\mathrm{C})$ is explicitly given by

$$
\sigma_{+, m}\left(\begin{array}{cc}
A & B \\
C & F
\end{array}\right)=\left(\begin{array}{cc}
A^{\dagger_{1}} & C^{\dagger_{3}} \\
B^{\dagger_{2}} & F^{\dagger_{4}}
\end{array}\right),
$$

where $A \in \operatorname{Mat}_{m \times m}(\mathcal{D}), B \in \operatorname{Mat}_{m \times n}(\mathcal{D}), C \in \operatorname{Mat}_{n \times m}(\mathcal{D})$, and $F \in \operatorname{Mat}_{n \times n}(\mathcal{D})$. And

$$
\mathcal{D}_{+, m}^{N}=\left\{\left(\begin{array}{cc}
A & B \\
-B^{\dagger_{2}} & F
\end{array}\right): A+A^{\dagger_{1}}=0 \text { and } F+F^{\dagger_{4}}=0\right\} .
$$

Observe that condition $\sigma_{+, m}(a)=-a$ implies $C^{\dagger_{3}}=-B$ and $B^{\dagger_{2}}=-C$, and these two conditions are equivalent since $\left(B^{\dagger_{2}}\right)^{\dagger_{3}}=B$. It is also possible to prove that $\mathcal{D}_{+, m}^{N}$ is a Lie subalgebra of $\mathcal{D}^{N}$ by direct computations, using that $\dagger_{1}$ and $\dagger_{4}$ are antiautomorphism, and the identities $B^{\dagger_{2}}$ $=t^{-1} B^{\dagger_{1}}, F^{\dagger_{4}}=t F^{\dagger_{3}},\left(B^{\dagger_{2}}\right)^{\dagger_{1}}=B t^{-1}$, etc. Observe that ${ }^{\dagger_{2}}$ and ${ }^{\dagger_{3}}$ are not antiautomorphism. The following identities are also useful

$$
\begin{gathered}
\dot{\sigma}_{ \pm, 0}\left(t^{-1} \dot{\sigma}_{ \pm, 0}(\cdot)\right)= \pm(\cdot) t^{-1}, \\
\dot{\sigma}_{ \pm,-1}\left(t^{-1} \dot{\sigma}_{ \pm, 0}(\cdot)\right)= \pm t^{-1}(\cdot),
\end{gathered}
$$

Case -:

Since the situation $N$ even and $m$ (also $n$ ) odd is impossible, we may suppose, due to the symmetry, that $n$ is even.

Now, consider the following maps on $\operatorname{Mat}_{m \times n}(\mathcal{D})$ :

$$
\begin{gathered}
A^{* 1}:=\dot{\sigma}_{-, 0}\left(A^{\dagger}\right), \\
B^{* 2}=\left(B_{1} \mid B_{2}\right)^{* 2}:=t^{-1} \dot{\sigma}_{-, 0}\left(\frac{-B_{2}^{\dagger}}{B_{1}^{\dagger}}\right), \\
C^{* 3}=\left(\frac{C_{1}}{C_{2}}\right)^{* 3}:=t \dot{\sigma}_{-,-1}\left(-C_{2}^{\dagger} \mid C_{1}^{\dagger}\right), \\
F^{* 4}=\left(\begin{array}{ll}
F_{1} & F_{2} \\
F_{3} & F_{4}
\end{array}\right)^{* 4}:=\dot{\sigma}_{-,-1}\left(\begin{array}{cc}
F_{4}^{\dagger} & -F_{2}^{\dagger} \\
-F_{3}^{\dagger} & F_{1}^{\dagger}
\end{array}\right),
\end{gathered}
$$

where $B_{i}$ are $m \times p$, with $n=2 p, C_{i}$ are $p \times m$, and $F_{i}$ are $p \times p$.

Then the anti-involution $\sigma_{-, m}$ on $\mathcal{D}^{N}$ is explicitly given by

$$
\sigma_{-, m}\left(\begin{array}{ll}
A & B \\
C & F
\end{array}\right)=\left(\begin{array}{ll}
A^{* 1} & C^{* 3} \\
B^{* 2} & F^{* 4}
\end{array}\right)
$$

where $A \in \operatorname{Mat}_{m \times m}(\mathcal{D}), B \in \operatorname{Mat}_{m \times n}(\mathcal{D}), C \in \operatorname{Mat}_{n \times m}(\mathcal{D})$, and $F \in \operatorname{Mat}_{n \times n}(\mathcal{D})$. And 


$$
\mathcal{D}_{-, m}^{N}=\left\{\left(\begin{array}{cc}
A & B \\
-B^{* 2} & F
\end{array}\right): A+A^{* 1}=0 \text { and } F+F^{* 4}=0\right\} .
$$

Note that we have again that condition $\sigma_{-, m}(a)=-a$ implies $C^{* 3}=-B$ and $B^{* 2}=-C$, and these two conditions are equivalent since $\left(B^{* 2}\right)^{* 3}=B$. Again, it is also possible to prove that $\mathcal{D}_{-, m}^{N}$ is a Lie subalgebra of $\mathcal{D}^{N}$ by direct computations, using that $*_{1}$ and $*_{4}$ are antiautomorphism. Observe that $*_{2}$ and $*_{3}$ are not antiautomorphism.

Observe that we may replace $\dagger$ by $T$ (usual transpose) in (3.6)-(3.8) and we get another family of involutions (denoted by $\sigma_{+, m}^{T}$ ) that do not preserve the principal Z-gradation, and the corresponding subalgebras are not Z-graded subalgebras of $\mathcal{D}^{N}$, but they are isomorphic to the others. Namely, using that $A^{\dagger}=J A^{T} J^{-1}$ where

$$
J=\left(\begin{array}{ccc}
0 & \cdots & 1 \\
\vdots & 1 & \vdots \\
1 & \cdots & 0
\end{array}\right)
$$

we get $\operatorname{Ad}_{J_{m, n}} \circ \sigma_{+, m}^{T}=\sigma_{+, m}$, where

$$
J_{m, n}=\left(\begin{array}{cc}
J_{m} & 0 \\
0 & J_{n}
\end{array}\right)
$$

and $J_{n}$ is the $n \times n$ matrix $J$. In the same way, we may replace $\dagger$ by $T$ in (3.10), getting another family of involutions denoted by $\sigma_{\bar{T}, m}^{T}$, and they produce subalgebras isomorphic to the others. More precisely, we have $\operatorname{Ad}_{J_{m, p, p}} \circ \sigma_{-, m}^{T}=\sigma_{-, m}$, where

$$
J_{m, p, p}=\left(\begin{array}{ccc}
J_{m} & 0 & 0 \\
0 & J_{p} & 0 \\
0 & 0 & J_{p}
\end{array}\right) .
$$

\section{GENERATORS OF $\mathcal{D}_{ \pm, m}^{N}$}

In this section we give a detailed description of the generators of $\mathcal{D}_{ \pm, m}^{N}$. Then we show that these subalgebras are simple Lie algebras.

Let us denote by $\mathrm{C}[w]^{(1)}$ the set of all odd polynomials in $\mathrm{C}[w]$, and by $\mathrm{C}[w]^{(0)}$ the set of all even polynomials in $C[w]$. And let $\bar{k}=0$ if $k$ is an odd integer and $\bar{k}=1$ if $k$ is even.

Note that $\mathcal{D}_{ \pm, m}^{N}=\left\{x-\sigma_{ \pm, m}(x): x \in \mathcal{D}^{N}\right\}$ and observe that by (3.5)

$$
\dot{\sigma}_{ \pm, b}\left(t^{k} f\left(D_{k}\right)\right)=( \pm t)^{k} f\left(-D_{k}\right),
$$

where $D_{k}=D+(k-b) / 2$. Therefore, by (3.6) and (3.10) the following is a set of generators of $\mathcal{D}_{ \pm, m}^{N}$.

From now on we will use the description of the elements in the subalgebras used in (3.8) and (3.12).

- Corresponding to the block $A$, that is $1 \leqslant i, j \leqslant m$ and $b=0\left(D_{k}=D+k / 2\right)$ :

First consider case + ,

for the case -

$$
\left\{t^{k}\left(f\left(D_{k}\right) E_{i, m+1-j}-f\left(-D_{k}\right) E_{j, m+1-i}\right): k \in \mathbb{Z}, f \in \mathrm{C}[x], 1 \leqslant i<j \leqslant m\right\}
$$

$$
\left\{t^{k}\left(f\left(D_{k}\right) E_{i, m+1-j}-(-1)^{k} f\left(-D_{k}\right) E_{j, m+1-i}\right): k \in \mathbb{Z}, f \in C[x], 1 \leqslant i<j \leqslant m\right\}
$$

and the generators on the opposite diagonal for the case + are

$$
\left\{t^{k} f\left(D_{k}\right) E_{i, m+1-i}: k \in \mathbb{Z}, f \in \mathbb{C}[x]^{(1)}, 1 \leqslant i \leqslant m\right\}
$$


and for the case - are

$$
\left\{t^{k} f\left(D_{k}\right) E_{i, m+1-i}: k \in \mathbb{Z}, f \in \mathrm{C}[x]^{(\bar{k})}, 1 \leqslant i \leqslant m\right\} .
$$

- Corresponding to the blocks $B-C(b=0)$ :

First consider case + . Here we have

$$
\left\{t^{k}\left(f\left(D_{k}\right) E_{i, m+j}-t^{-1} f\left(-D_{k}\right) E_{N+1-j, m+1-i}\right): k \in \mathbb{Z}, f \in \mathbb{C}[x], 1 \leqslant i \leqslant m \text {, and } 1 \leqslant j \leqslant N-m\right\} .
$$

And for the case -,

$$
\begin{aligned}
& \left\{t^{k}\left(f\left(D_{k}\right) E_{i, m+j}-(-1)^{k} t^{-1} f\left(-D_{k}\right) E_{N+1-j, m+1-i}\right): k \in \mathbb{Z}, f \in \mathbb{C}[x], 1 \leqslant i \leqslant m,\right. \\
& \quad \text { and } 1 \leqslant j \leqslant p\}, \\
& \left\{t^{k}\left(f\left(D_{k}\right) E_{i, m+j}+(-1)^{k} t^{-1} f\left(-D_{k}\right) E_{N+1-j, m+1-i}\right): k \in \mathbb{Z}, f \in \mathbb{C}[x], 1 \leqslant i \leqslant m,\right. \\
& \quad \text { and } p+1 \leqslant j \leqslant N-m\} .
\end{aligned}
$$

- Corresponding to the blocks $F\left(b=-1\right.$ and $\left.D_{k}=D+(k+1) / 2\right)$ :

Case +:

$$
\left\{t^{k}\left(f\left(D_{k}\right) E_{m+i, N+1-j}-f\left(-D_{k}\right) E_{m+j, N+1-i}\right): k \in \mathbb{Z}, \quad f \in \mathbb{C}[x], 1 \leqslant i<j \leqslant N-m\right\}
$$

and the generators on the opposite diagonal are

$$
\left\{t^{k} f\left(D_{k}\right) E_{m+i, N+1-i}: k \in \mathbb{Z}, f \in \mathbb{C}[x]^{(1)}, 1 \leqslant i \leqslant N-m\right\} .
$$

For the case - they are

$$
\begin{array}{r}
\left\{t^{k}\left(f\left(D_{k}\right) E_{m+i, m+j}-(-1)^{k} f\left(-D_{k}\right) E_{N+1-j, N+1-i}\right): k \in \mathbb{Z}, f \in \mathbb{C}[x], 1 \leqslant i, j \leqslant p\right\}, \\
\left\{t^{k}\left(f\left(D_{k}\right) E_{m+i, N+1-j}+(-1)^{k} f\left(-D_{k}\right) E_{m+j, N+1-i}\right): k \in \mathbb{Z}, f \in \mathbb{C}[x], 1 \leqslant i<j \leqslant p\right\}, \\
\left\{t^{k}\left(f\left(D_{k}\right) E_{N+1-i, m+j}+(-1)^{k} f\left(-D_{k}\right) E_{N+1-j, m+i}\right): k \in \mathbb{Z}, f \in \mathbb{C}[x], 1 \leqslant i<j \leqslant p\right\}
\end{array}
$$

and the generators on the opposite diagonal are

$$
\left\{t^{k} f\left(D_{k}\right) E_{m+i, N+1-i}: k \in \mathbb{Z}, \quad f \in \mathbb{C}[x]^{(\overline{k+1})}, 1 \leqslant i \leqslant N-m\right\} .
$$

Using the above-given description we can prove the following

Theorem 2: The Lie algebras $\mathcal{D}_{ \pm, m}^{N}$ are simple.

Since this is a rather technical proof, we refer to the Appendix.

\section{GEOMETRIC REALIZATION OF $\sigma_{ \pm, m}$}

In this section we give a geometric realization of $\sigma_{ \pm, f, m}$, for arbitrary $f$. The algebra $\mathcal{D}^{N}$ acts on the space $V=\mathrm{C}^{N}\left[t, t^{-1}\right]$ and one has two bilinear forms on $V$ :

$$
B_{ \pm}(h, g)=\operatorname{Res}_{t} h^{T} J_{ \pm} g,
$$

where

$$
J_{+}=\left(\begin{array}{cc}
t^{-f-1} J_{m} & 0 \\
0 & t^{-f} J_{n}
\end{array}\right), \quad J_{-}=\left(\begin{array}{ccc}
t^{-f-1} J_{m} & 0 & 0 \\
0 & 0 & t^{-f} J_{p} \\
0 & -t^{-f} J_{p} & 0
\end{array}\right) \circ \Phi
$$

with $\Phi: V \rightarrow V$ given by $\Phi(h(t))=h(-t), h(t) \in V$, and $J_{m}$ as in (3.13). Observe that $V$ $=\mathbb{C}^{m}\left[t, t^{-1}\right] \times \mathbb{C}^{n}\left[t, t^{-1}\right]$ is an orthogonal decomposition of $V$. Now, consider the following proposition. 
Proposition 1: (a) The bilinear forms $B_{ \pm}$are nondegenerated and $B_{+}$is symmetric. If $f$ is even (respectively, odd) then $B_{-}$is symmetric (respectively, skew-symmetric).

(b) For any $L \in \mathcal{D}^{N}$ and $h, g \in V$ we have

$$
B_{ \pm}(L h, g)=B_{ \pm}\left(h, \sigma_{ \pm, f, m}(L) g\right),
$$

that is, $L$ and $\sigma_{ \pm, f, m}(L)$ are adjoint operators with respect to $B_{ \pm}$.

Proof: (a) The statements that $B_{ \pm}$are nondegenerated and $B_{+}$is symmetric are straightforward. Let us see $B_{-}$:

$$
\begin{aligned}
& B_{-}\left(t^{k} e_{i}, t^{l} e_{j}\right)=\operatorname{Res}_{t} e_{i}^{T}\left(\begin{array}{ccc}
(-1)^{l} t^{k+1-f-1} J_{m} & 0 & 0 \\
0 & 0 & (-1)^{l} t^{k+l-f} J_{p} \\
0 & (-1)^{l} t^{k+l-f}\left(-J_{p}\right) & 0
\end{array}\right) e_{j} \\
& =e_{i}^{T}\left(\begin{array}{ccc}
(-1)^{l} \delta_{k+l-f, 0} J_{m} & 0 & 0 \\
0 & 0 & (-1)^{l} \delta_{k+l-f,-1} J_{p} \\
0 & (-1)^{l} \delta_{k+l-f,-1}\left(-J_{p}\right) & 0
\end{array}\right) e_{j} \\
& =(-1)^{f} e_{j}^{T}\left(\begin{array}{ccc}
(-1)^{k} \delta_{k+l-f, 0} J_{m} & 0 & 0 \\
0 & 0 & (-1)^{k} \delta_{k+l-f,-1} J_{p} \\
0 & (-1)^{k} \delta_{k+l-f,-1}\left(-J_{p}\right) & 0
\end{array}\right) e_{i} \\
& =(-1)^{f} B_{-}\left(t^{l} e_{j}, t^{k} e_{i}\right) .
\end{aligned}
$$

Therefore, if $f$ is even $B_{-}$is symmetric and if $f$ is odd $B_{-}$is skew-symmetric.

(b) Let $L=t^{k} p(D)\left(\begin{array}{ll}A & B \\ C & F\end{array}\right), h=t^{r} e_{p}$, and $g=t^{s} e_{q}$ be as shown previously. We will consider only the + case. The - case is completely analogous being careful with the definition of $J_{-}$. Then recall that

$$
\sigma_{+, m, f}(L)(g)=t^{s+k}\left(\begin{array}{cc}
p(-k-s+f) A^{\dagger} & t p(-k-s+f-1) C^{\dagger} \\
t^{-1} p(-k-s+f) B^{\dagger} & p(-k-s+f-1) F^{\dagger}
\end{array}\right) e^{q}
$$

and $L(h)=t^{k+r} p(r)\left(\begin{array}{ll}A B & B\end{array}\right) e_{p}$. Let us compute,

$$
\begin{aligned}
B_{+}\left(L\left(t^{r} e_{p}\right), t^{s} e_{q}\right) & =\operatorname{Res}_{t} t^{k+r} p(r) e_{p}^{T}\left(\begin{array}{cc}
A & B \\
C & F
\end{array}\right)^{T}\left(\begin{array}{cc}
t^{-f-1} J_{m} & 0 \\
0 & t^{-f} J_{n}
\end{array}\right) t^{s} e_{q} \\
& =(p, q) \text { entry } p(r)\left(\begin{array}{cc}
\delta_{k+r+s-f, 0} A^{T} J_{m} & \delta_{k+r+s-f,-1} C^{T} J_{n} \\
\delta_{k+r+s-f, 0} B^{T} J_{m} & \delta_{k+r+s-f,-1} F^{T} J_{n}
\end{array}\right) .
\end{aligned}
$$

On the other hand, we have

$$
\begin{aligned}
B_{+}\left(h, \sigma_{+, f, m}(L) g\right)= & \operatorname{Res}_{t} t^{r+s+k} e_{p}^{T}\left(\begin{array}{cc}
t^{-f-1} J_{m} 0 & \\
0 & t^{-f} J_{n}
\end{array}\right) \\
& \cdot\left(\begin{array}{cc}
p(-k-s+f) A^{\dagger} & t p(-k-s+f-1) C^{\dagger} \\
t^{-1} p(-k-s+f) B^{\dagger} & p(-k-s+f-1) F^{\dagger}
\end{array}\right) e_{q} \\
= & \left(\begin{array}{cc}
\delta_{k+r+s-f, 0} p(-k-s+f) J_{m} A^{\dagger} & \delta_{k+r+s-f,-1} p(-k-s+f-1) J_{m} C^{\dagger} \\
\delta_{k+r+s-f, 0} p(-k-s+f) J_{n} B^{\dagger} & \delta_{k+r+s-f,-1} p(-k-s+f-1) J_{n} F^{\dagger}
\end{array}\right)_{p, q} .
\end{aligned}
$$


Now, comparing (5.3) with (5.4) we finish the proof.

Remark 2: Analogously, we can define the following nondegenerate bilinear forms on $V$ (in this case we consider $f=0$ for simplicity):

$$
B_{ \pm}^{T}(h, q)=\operatorname{Res}_{t} h^{T} J_{ \pm, T} g,
$$

where

$$
J_{+, T}=\left(\begin{array}{cc}
t^{-1} I_{m} & 0 \\
0 & I_{n}
\end{array}\right), \quad J_{-, T}=\left(\begin{array}{ccc}
t^{-1} I_{m} & 0 & 0 \\
0 & 0 & I_{p} \\
0 & -I_{p} & 0
\end{array}\right) \circ \Phi
$$

with $I_{m}$ the $m \times m$ identity matrix, and it is easy to see that they satisfy

$$
B_{ \pm}(L h, g)=B_{ \pm}\left(h, \sigma_{ \pm, m}^{T}(L) g\right),
$$

where $\sigma_{ \pm, m}^{T}$ were defined in (3.13). Therefore, we can claim that $\mathcal{D}_{+, m}^{N}$ is a subalgebra of $\mathcal{D}^{N}$ of type $o(m, n)$ and $\mathcal{D}_{-, m}^{N}$ is a subalgebra of $\mathcal{D}^{N}$ of type $\operatorname{ssp}(m, n)$ (orthogonal-symplectic).

\section{CONFORMAL ALGEBRA ASSOCIATED WITH $\mathcal{D}_{+, m}^{N}$}

In this section we will study the conformal algebra associated with $\mathcal{D}_{+, m}^{N}$. We will follow the notation on Ref. 2. Recall that $\mathcal{D}^{N}=\mathcal{D}^{1} \otimes_{\mathrm{C}} \mathrm{Mat}_{N} \mathrm{C}$, viewed as an associative algebra is a formal distribution algebra with the family of pairwise local formal distributions

$$
\mathcal{F}=\left\{J_{A}^{n}(z)=J^{n}(z) \otimes A: n \in \mathbb{Z}_{+}, A \in \operatorname{Mat}_{N} \mathrm{C}\right\},
$$

where $J^{n}(z)=\Sigma_{j \in \mathbb{Z}} t^{j}\left(-\partial_{t}\right)^{n} z^{-j-1}=\delta(t-z)\left(-\partial_{t}\right)^{n}$, cf. Ref. 2, example 2.10. Recall that given a collection of mutually local formal distributions $\mathcal{F}$, the closure $\overline{\mathcal{F}}$ is defined as the minimal $\mathrm{C}[\partial]$-module closed under all $n$th products, $n \in \mathbb{Z}_{+}$(see Ref. 2, pp. 39).

The associated associative conformal algebra is

$$
\operatorname{Cend}_{N}=\oplus_{n \in Z_{+}} \mathrm{C}[\partial] J^{n} \otimes \mathrm{Mat}_{N} \mathrm{C}
$$

with $\lambda$-product

$$
J_{A \lambda}^{k} J_{B}^{l}=\sum_{j=0}^{k}\left(\begin{array}{l}
k \\
j
\end{array}\right)(\lambda+\partial)^{j} J_{A B}^{k+l-j} .
$$

We will denote by $g c_{N}$ the conformal (associative) algebra Cend ${ }_{N}$ viewed as a Lie conformal algebra with the $\lambda$-bracket

$$
\left[J_{A \lambda}^{k} J_{B}^{l}\right]=\sum_{j=0}^{k}\left(\begin{array}{l}
k \\
j
\end{array}\right)(\lambda+\partial)^{j} J_{A B}^{k+l-j}-\sum_{j=0}^{l}\left(\begin{array}{l}
l \\
j
\end{array}\right)(-\lambda)^{j} J_{B A}^{k+l-j} .
$$

For simplicity, we will introduce the following bijective map that we learn from Kac's lectures at MIT (Spring'98), called the symbol,

$$
\begin{gathered}
\text { Symb: } g c_{N} \rightarrow \operatorname{Mat}_{N}[\partial, x], \\
\sum_{k} A_{k}(\partial) J^{k} \mapsto \sum_{k} A_{k}(\partial) x^{k},
\end{gathered}
$$

where $A_{k}(\partial) \in \operatorname{Mat}_{N}(C[\partial])$. The transferred $\lambda$-bracket is 


$$
A(\partial, x)_{\lambda} B(\partial, x)=A(-\lambda, x+\lambda+\partial) B(\lambda+\partial, x)-B(\lambda+\partial,-\lambda+x) A(-\lambda, x) .
$$

Now, we will try to extend the anti-involutions $\sigma_{+, m}$ on $\mathcal{D}^{N}$ to the conformal algebra $\operatorname{Cend}_{N}$ (here we return to our normalization $f=0$ made in Sec. III). When we apply $\sigma_{+, m}$ to the fields $J_{A}^{k}$ we find some problems. In order to show this we will need the following formulas:

$$
\dot{\sigma}_{+, 0}\left(\partial_{t}\right)=-t \partial_{t} t^{-1}, \quad \dot{\sigma}_{+,-1}\left(\partial_{t}\right)=-\partial_{t}
$$

and

$$
\dot{\sigma}_{+, b}(\delta(t-z))=\delta(t-z) \text { for any } b .
$$

If $1 \leqslant i, j \leqslant m$, using (3.6):

$$
\begin{aligned}
\sigma_{+, m}\left(J_{E_{i j}}^{1}(z)\right) & =\sigma_{+, m}\left(\delta(t-z)\left(-\partial_{t}\right) E_{i j}\right) \\
& =\dot{\sigma}_{+, 0}\left(\delta(t-z)\left(-\partial_{t}\right)\right) E_{m+1-j, m+1-i} \\
& =\dot{\sigma}_{+, 0}\left(-\partial_{t}\right) \dot{\sigma}_{+, 0}(\delta(t-z)) E_{m+1-j, m+1-i} \\
& =t \partial_{t} t^{-1} \delta(t-z) E_{m+1-j, m+1-i} \\
& =\left(\delta(t-z) \partial_{t}+t\left(t^{-1} \delta(t-z)\right)^{\prime}\right) E_{m+1-j, m+1-i} \\
& =\left(\delta(t-z) \partial_{t}-t^{-1} \delta(t-z)+\delta_{t}^{\prime}(t-z)\right) E_{m+1-j, m+1-i} \\
& =-J_{E_{m+1-j, m+1-i}^{1}}(z)-t^{-1} J_{E_{m+1-j, m+1-i}^{0}}(z)-\partial_{z} J_{E_{m+1-j, m+1-i}}(z)
\end{aligned}
$$

Warning: The second term in the last line of (6.4) has an extra $t^{-1}$.

And if $1 \leqslant i, j \leqslant N-m$,

$$
\begin{aligned}
\sigma_{+, m}\left(J_{E_{m+i, m+j}}^{1}(z)\right) & =\dot{\sigma}_{+,-1}\left(\delta(t-z)\left(-\partial_{t}\right)\right) E_{N+1-j, N+1-i} \\
& =\partial_{t} \delta(t-z) E_{N+1-j, N+1-i} \\
& =\left(\delta(t-z) \partial_{t}+\delta_{t}^{\prime}(t-z)\right) E_{N+1-j, N+1-i} \\
& =-J_{E_{N+1-j, N+1-i}}^{1}(z)-\partial_{z} J_{E_{N+1-j, N+1-i}}^{0}(z) .
\end{aligned}
$$

In the same way, for $1 \leqslant i \leqslant m$ and $1 \leqslant j \leqslant N-m$ we have

$$
\sigma_{+, m}\left(J_{E_{i, m+j}}^{1}(z)\right)=-t^{-1}\left(J_{E_{N+1-j, m+1-i}}^{1}(z)+\partial_{z} J_{E_{N+1-j, m+1-i}}^{0}(z)\right) .
$$

and for $1 \leqslant i \leqslant N-m$ and $1 \leqslant j \leqslant m$, we get

$$
\sigma_{+, m}\left(J_{E_{m+i, j}}^{1}(z)\right)=-t\left(J_{E_{m+1-j, N+1-i}^{1}}(z)+\partial_{z} J_{E_{m+1-j, N+1-i}}^{0}(z)\right)-J_{E_{m+1-j, N+1-i}}^{0}(z) .
$$

Remark 3: (a) From (6.4) and (6.6) to (6.7), we observe that

$$
\sigma_{+, m}(\mathcal{F}) \oplus \overline{\mathcal{F}}
$$

We will return to this point later.

(b) From (6.5) and using symbol, we have that 


$$
\sigma_{+, m}\left(\begin{array}{cc}
0 & 0 \\
0 & F(\partial, x)
\end{array}\right)=\left(\begin{array}{cc}
0 & 0 \\
0 & F^{\dagger}(\partial,-\partial-x)
\end{array}\right)
$$

So if we consider the degenerated case $m=0$, we have $\sigma_{+, 0}(A)=\dot{\sigma}_{+,-1}\left(A^{\dagger}\right)$ getting in this way an anti-involution on $g c_{N}$. In terms of symbol, the corresponding conformal subalgebra fixed by $-\sigma_{+, 0}$, which is usually denoted by $o c_{N}$, is the linear C-span of

$$
\overline{A(\partial, x)}:=A(\partial, x)-\sigma_{+, 0}(A(\partial, x))=A(\partial, x)-A(\partial,-\partial-x)
$$

with $A \in \mathrm{Mat}_{N}$ and the corresponding $\lambda$-bracket given by

$$
\begin{aligned}
{\left[\overline{A(\partial, x)_{\lambda}} \overline{B(\partial, x)}\right]=} & \overline{A(-\lambda, \lambda+\partial+x) B(\lambda+\partial, x)}-\overline{B(\lambda+\partial,-\lambda+x) A(-\lambda,-\lambda+x)} \\
& -\overline{A^{\dagger}(-\lambda,-\partial-x) B(\lambda+\partial, x)}+\overline{B(\lambda+\partial,-\lambda+x) A^{\dagger}(-\lambda, \lambda-x)} .
\end{aligned}
$$

(c) In the case $N=1$ we have the same picture as in Ref. 1 , where the fields $W^{n}(w):=\delta(t$ $-z)\left(-\partial_{t}\right)^{n}+(-1)^{n+1}\left(-\partial_{t}\right)^{n} \delta(t-z)$, are introduced. Transferred in terms of symbol, $w^{n}=x^{n}$ $-(-\partial-x)^{n}$ and the $\lambda$-bracket is simply (cf. Ref. 1, pp. 131-132)

$$
\left[w_{\lambda}^{n} w^{m}\right]=(-\lambda-\partial-w)^{n} w^{m}-(\partial-w)^{m} w^{n}-(w+\partial)^{n} w^{m}+(\lambda-w)^{m}(-\lambda+w)^{n} .
$$

Let us return to Remark 3(a). Consider the Lie algebra of matrix differential operators with constant coefficients $\mathfrak{g}:=\mathrm{Mat}_{N} \mathrm{C}[\partial]$.

Now, for each $0 \leqslant m \leqslant N$, we have a $Z_{2}$-gradation in $\mathfrak{g}=\mathfrak{g}_{0} \oplus \mathfrak{g}_{1}$ where,

$$
\begin{gathered}
\mathfrak{g}_{0}=\left(\begin{array}{cc}
\operatorname{Mat}_{m} \mathrm{C}[\partial] & 0 \\
0 & \mathrm{Mat}_{N-m} \mathrm{C}[\partial]
\end{array}\right), \\
\mathfrak{g}_{1}=\left(\begin{array}{cc}
0 & \operatorname{Mat}_{m \times N-m} \mathrm{C}[\partial] \\
\operatorname{Mat}_{N-m \times m} \mathrm{C}[\partial] & 0
\end{array}\right) .
\end{gathered}
$$

Let us consider the Lie algebra $\mathcal{D}^{N, 1 / 2}$ of matrix differential operators with coefficients in $\mathrm{C}\left[t^{1 / 2}, t^{-1 / 2}\right]$, which can be seen as

$$
\mathcal{D}^{N, 1 / 2}=\mathrm{C}\left[t^{1 / 2}, t^{-1 / 2}\right] \otimes \mathfrak{g} .
$$

Consider the following subalgebra of $\mathcal{D}^{N, 1 / 2}$ :

$$
\mathcal{D}_{m}^{N, 1 / 2}=\mathrm{C}\left[t^{1}, t^{-1}\right] \otimes \mathfrak{g}_{0} \oplus t^{1 / 2} \mathrm{C}\left[t^{1}, t^{-1}\right] \otimes \mathfrak{g}_{1} .
$$

This is a twisted $\mathcal{D}^{N}$ algebra. Let us define the isomorphism $T_{m}: \mathcal{D}^{N, 1 / 2} \rightarrow \mathcal{D}^{N, 1 / 2}$ given by

$$
T_{m}(A)=\left(\begin{array}{cc}
t^{-1 / 2} I_{m} & 0 \\
0 & I_{N-m}
\end{array}\right) A\left(\begin{array}{cc}
t^{1 / 2} I_{m} & 0 \\
0 & I_{N-m}
\end{array}\right) .
$$

Note that $T_{m}$ restricted to $\mathcal{D}^{N}$ gives us an isomorphism between $\mathcal{D}^{N}$ and $\mathcal{D}_{m}^{N, 1 / 2}$. Via this isomorphism we translate $\sigma_{+, m}$ to $\mathcal{D}_{m}^{N, 1 / 2}$, getting $T_{m}{ }^{\circ} \sigma_{+, m}{ }^{\circ} T_{m}^{-1}=\sigma_{*}$ where

$$
\sigma_{*}\left(\begin{array}{cc}
A & B \\
C & F
\end{array}\right)=\dot{\sigma}_{+,-1}\left(\begin{array}{cc}
A^{\dagger} & C^{\dagger} \\
B^{\dagger} & F^{\dagger}
\end{array}\right) .
$$

Observe that $\sigma_{*}$ is equivalent to the involution defining $o c_{N}$ extended to $\mathcal{D}_{m}^{N, 1 / 2}$. Take the subalgebra $\mathcal{D}_{m, \sigma_{*}}^{N, 1 / 2}$ of $\mathcal{D}_{m}^{N, 1 / 2}$ fixed by $-\sigma_{*}$. This is isomorphic to $\mathcal{D}_{+, m}^{N}$. 
Following the notation in Ref. $3, \mathcal{D}_{m}^{N, 1 / 2}$ gives rise to $1 / 2 Z$-twisted formal distributions algebra with local family

$$
\mathcal{F}_{m}=\left\{J_{A_{i}}^{n, i / 2}(z):=J^{n, i / 2}(z) \otimes A_{i}:=\sum_{k \in Z} t^{k+i / 2}\left(-\partial_{t}\right)^{n} z^{-k-1-i / 2} A_{i}: A_{i} \in\left(\operatorname{Mat}_{N} \mathrm{C}\right)_{i}, \quad i \in \mathbb{Z}_{2}\right\}
$$

where

$$
\begin{gathered}
\left(\mathrm{Mat}_{N} \mathrm{C}\right)_{0}=\left\{\left(\begin{array}{cc}
A & 0 \\
0 & F
\end{array}\right): A \in \operatorname{Mat}_{m} \mathrm{C}, F \in \mathrm{Mat}_{N-m} \mathrm{C}\right\}, \\
\left(\mathrm{Mat}_{N} \mathrm{C}\right)_{1}=\left\{\left(\begin{array}{cc}
0 & B \\
C & 0
\end{array}\right): B \in \mathrm{Mat}_{m \times N-m} \mathrm{C}, C \in \mathrm{Mat}_{N-m \times m} \mathrm{C}\right\} .
\end{gathered}
$$

Observe that $J_{A_{i}}^{n, i / 2}(z)=\delta_{i / 2}(z-t)(-\partial)^{n} A_{i}$, where $\delta_{i / 2}(z-t)=\Sigma_{k \in \mathbb{Z}} t^{k+i / 2} z^{-k-1-i / 2}$.

Define $\operatorname{symb}\left(J_{A_{i}}^{n, i / 2}\right)=x A_{i}$ where $A_{i} \in \operatorname{Mat}_{N}(\mathbb{C})_{i}, i \in\{0,1\}$. Under this identification, the associated $\mathbb{Z}_{2}$-graded conformal algebra is

$$
g c_{N, m}:=\left(\operatorname{Mat}_{N} \mathrm{C}[\partial, x]\right)_{0} \oplus\left(\operatorname{Mat}_{N} \mathrm{C}[\partial, x]\right)_{1}
$$

with $\lambda$-product

$$
\left[A_{i}(\partial, x)_{\lambda} B_{j}(\partial, x)\right]=A_{i}(-\lambda, \lambda+\partial+x) B_{j}(\lambda+\partial, x)-B_{j}(\lambda+\partial,-\lambda+x) A_{i}(-\lambda, x) .
$$

Therefore, $g c_{N, m}$ is simply $g c_{N}=\operatorname{Mat}_{N}(\mathbb{C}[\partial, x])$ endowed with a $\mathbb{Z}_{2}$-gradation that comes from the $\mathbb{Z}_{2}$-gradation in $\mathrm{Mat}_{N}(\mathrm{C})$ given by (6.8).

It is easy to see that $\sigma_{*}\left(\mathcal{F}_{m}\right) \subset \overline{\mathcal{F}_{m}}$, getting in this way the $\mathbb{Z}_{2}$-graded conformal subalgebra $o c_{N, m}$ of $g c_{N, m}$ fixed by $-\sigma_{*}$. Obviously, we can see $o c_{N, m}$ as $o c_{N}$ with a $Z_{2}$-gradation, or as the $Z_{2}$-graded conformal algebra associated with the $1 / 2 Z$-twisted formal distribution algebra $\mathcal{D}_{m, \sigma}^{N, 1 / 2}$.

\section{CONFORMAL ALGEBRA ASSOCIATED WITH $\mathcal{D}_{-, m}^{N}$}

In this section we will study the conformal algebra associated with $\mathcal{D}_{-, m}^{N}$. As in Sec. VI we will follow the notation in Refs. 2 and 3. Recall that in the + case, we first considered the degenerated case $\sigma_{+, 0}$ (that is $m=0$ ) where we obtained a formal distribution algebra structure on $\mathcal{D}_{+, 0}^{N}$, but in the case $1 \leqslant m<N$ we obtained a 1/2Z-twisted formal distribution algebra. Similarly, in the case -, we will first consider the degenerated case $m=N$. Here we are forced to take the Lie algebra $\mathcal{D}_{-, N}^{N}$ as $Z_{2}$-local formal distribution algebra, and in the case $1 \leqslant m<N$ we get a double structure, that is $1 / 2 \mathrm{Z}$-twisted and $\mathrm{Z}_{2}$-local formal distribution algebra.

Let $m=N$. Here $\sigma_{-, N}(A)=\dot{\sigma}_{-, 0}\left(A^{\dagger}\right)$. In the case of $N=1$ we get the Lie algebra $\mathcal{D}^{-}$considered in Ref. 1. In this paper as in our case the most convenient choice is the isomorphic subalgebra $\mathcal{D}_{-}^{N}$ which is the subalgebra of $\mathcal{D}^{N}$ fixed by $-\sigma_{-}:=-\dot{\sigma}_{-,-1}\left(A^{\dagger}\right)$ (because $\dot{\sigma}_{-,-1}\left(\partial_{t}\right)=\partial_{t}$ and $\left.\dot{\sigma}_{-,-1}(t)=-t\right)$.

Let us consider as before the fields $J_{A}^{n}(z) \in \mathcal{F}$ [cf. (6.1)]. Therefore, we have that

$$
\sigma_{-}\left(J_{A}^{n}(z)\right)=\sigma_{-}\left(\delta(z-t)\left(-\partial_{t}\right)^{n} A\right)=\left(-\partial_{t}\right)^{n} \delta(z+t) A^{\dagger}
$$

with $\delta(z+t)=z^{-1} \Sigma_{k \in \mathbb{Z}}(-t / z)^{k}$. This motivates one to consider the family

$$
\mathcal{F}^{ \pm}=\left\{J_{A}^{n, \pm 1}(z):=\delta(z \pm t)\left(-\partial_{t}\right)^{n} A: n \in \mathbb{Z}_{+} \text {and } A \in \mathrm{Mat}_{n} \mathrm{C}\right\},
$$

which is a family of pairwise $\mathbb{Z}_{2}$-local formal distributions, (here $\left.\mathbb{Z}_{2}=\{1,-1\}\right)$, with OPE $(\alpha, \beta$ $\left.\in \mathbb{Z}_{2}\right)$ : 


$$
\begin{aligned}
{\left[J_{A}^{m, \alpha}(z), J_{B}^{n, \beta}(w)\right]=} & \sum_{k=0}^{m} \sum_{r=0}^{k}\left(\begin{array}{c}
m \\
k
\end{array}\right)\left(\begin{array}{c}
k \\
r
\end{array}\right) \beta^{k}\left(\partial_{w}^{r} J_{A B}^{m+n-k, \beta}(w)\right) \partial_{w}^{k-r} \delta\left(z-\alpha \beta^{-1} w\right) \\
& -\sum_{l=0}^{n}\left(\begin{array}{c}
n \\
l
\end{array}\right)(-\beta)^{l} J_{B A}^{m+n-l, \beta}(w) \partial_{w}^{l} \delta\left(z-\alpha \beta^{-1} w\right) .
\end{aligned}
$$

As in Sec. VII in Ref. 3, we introduce the following operator on the space of formal distributions: $T_{\alpha}(a(z))=\alpha a(\alpha z)$, with $\alpha \in \mathbb{Z}_{2}$. Note that $\mathcal{F}^{ \pm}$is closed under all $T_{\alpha}$. Therefore, if we take $\operatorname{symb}\left(J_{A}^{1,1}\right)=x A$ and $\operatorname{symb}\left(J_{A}^{1,-1}\right)=y A$, then $\left(\mathcal{D}_{-}^{N}, \mathcal{F}^{ \pm}\right)$gives rise to a conformal algebra

$$
R=\overline{\mathcal{F}^{ \pm}} \simeq g c_{N} \oplus g c_{N}=\operatorname{Mat}_{N}(\mathrm{C}[\partial, x]) \oplus \operatorname{Mat}_{N}(\mathrm{C}[\partial, y]),
$$

since the $\lambda$-bracket is

$$
\begin{aligned}
& {\left[A(\partial, x)_{\lambda} B(\partial, x)\right]=A(-\lambda, \lambda+\partial+x) B(\lambda+\partial, x)-B(\lambda+\partial,-\lambda+x) A(-\lambda, x),} \\
& {\left[A(\partial, y)_{\lambda} B(\partial, y)\right]=A(-\lambda,-\lambda-\partial+y) B(\lambda+\partial, y)-B(\lambda+\partial, \lambda+y) A(-\lambda, y),}
\end{aligned}
$$

and $\left[A(\partial, x)_{\lambda} B(\partial, y)\right]=0$. The $\mathbb{Z}_{2}$-action on it by semilinear automorphisms (this is $\alpha T_{\alpha} \partial=\partial T_{\alpha}$ and $\left.T_{\alpha}\left(\left[x_{\lambda} y\right]\right)=\left[T_{\alpha}(x)_{\alpha \lambda} T_{\alpha}(y)\right]\right)$, is given by $T_{-1}(A(\partial, x))=A(\partial, y)$ and $T_{-1}(A(\partial, y))$ $=A(\partial, x)$.

Now, we have that

$$
\begin{aligned}
& \sigma_{-}(A(\partial, x))=A^{\dagger}(\partial, y-\partial), \\
& \sigma_{-}(A(\partial, y))=A^{\dagger}(\partial, x+\partial) .
\end{aligned}
$$

Thus, the subalgebra fixed by $-\sigma_{-}$is the C-linear span of $\overline{A(\partial, x)}:=A(\partial, x)-A^{\dagger}(\partial, y-\partial)$, (observe that $\overline{A(\partial, y)}=-\overline{A(\partial, x+\partial)})$.

The $\lambda$-bracket is

$$
\left[\overline{A(\partial, x)_{\lambda} B(\partial, x)}\right]=\overline{A(-\lambda, x+\lambda+\partial) B(\lambda+\partial, x)}-\overline{B(\lambda+\partial,-\lambda+x) A(-\lambda, x)} .
$$

Note that it is easy to show that

$$
T_{-1}(\overline{A(\partial, x)})=-\left(\overline{A^{\dagger}(-\partial, x+\partial)}\right) .
$$

Therefore, the associated conformal subalgebra is isomorphic to $g c_{N}$ with a $Z_{2}$-action by semilinear automorphisms given by $T_{1}=i d$ and $T_{-1}$.

Remark 4: Observe that we can collapse to the case $N=1$, getting $g c_{1}$ as the $\mathbb{Z}_{2}$-conformal algebra associated with the Lie algebra $\mathcal{D}_{-}$in Ref. 1.

Now, let us consider $1 \leqslant m<N$. In this case we need to combine the $1 / 2 Z$-twisted and the $Z_{2}$-local notions.

As in Sec. VI, consider the Lie algebra of matrix differential operators with constant coefficients $\mathfrak{g}:=\mathrm{Mat}_{N} \mathrm{C}[\partial]$. For each $0 \leqslant m \leqslant N$, we take the $\mathbb{Z}_{2}$-gradation in $\mathfrak{g}=\mathfrak{g}_{0} \oplus \mathfrak{g}_{1}$ as before.

Let us consider the Lie algebra $\mathcal{D}^{N, 1 / 2}$ of matrix differential operators with coefficients in $\mathrm{C}\left[t^{1 / 2}, t^{-1 / 2}\right]$, and consider the following subalgebra:

$$
\mathcal{D}_{m}^{N, 1 / 2}=\mathbb{C}\left[t^{1}, t^{-1}\right] \otimes \mathfrak{g}_{0} \oplus t^{1 / 2} \mathbb{C}\left[t^{1}, t^{-1}\right] \otimes \mathfrak{g}_{1} .
$$

This is a twisted $\mathcal{D}^{N}$ algebra. Let us define the isomorphism $T_{m}: \mathcal{D}^{N, 1 / 2} \rightarrow \mathcal{D}^{N, 1 / 2}$ given by 


$$
T_{m}(A)=\left(\begin{array}{cc}
\sqrt{i} t^{-1 / 2} I_{m} & 0 \\
0 & I_{N-m}
\end{array}\right) A\left(\begin{array}{cc}
\frac{1}{\sqrt{i}} t^{1 / 2} I_{m} & 0 \\
0 & I_{N-m}
\end{array}\right) .
$$

Note that $T_{m}$ restricted to $\mathcal{D}^{N}$ gives us an isomorphism between $\mathcal{D}^{N}$ and $\mathcal{D}_{m}^{N, 1 / 2}$. Via this isomorphism we translate $\sigma_{-, m}$ to $\mathcal{D}_{m}^{N, 1 / 2}$, getting $T_{m} \circ \sigma_{-, m}^{\circ} T_{m}^{-1}=\sigma_{*}$ where

$$
\sigma_{*}(A)=\hat{\sigma}_{-,-1} A^{\ddagger},
$$

with $A \in \mathrm{Mat}_{N} \mathrm{C}$ and

$$
A^{\ddagger}=\left(\begin{array}{lll}
A_{1} & B_{1} & B_{2} \\
C_{1} & F_{1} & F_{2} \\
C_{2} & F_{3} & F_{4}
\end{array}\right)^{\ddagger}=\left(\begin{array}{ccc}
A_{1}^{\dagger} & -C_{2}^{\dagger} & C_{1}^{\dagger} \\
-B_{2}^{\dagger} & F_{4}^{\dagger} & -F_{2}^{\dagger} \\
B_{1}^{\dagger} & -F_{3}^{\dagger} & F_{1}^{\dagger}
\end{array}\right) .
$$

Take the subalgebra $\mathcal{D}_{m, \sigma_{*}}^{N, 1 / 2}$ of $\mathcal{D}_{m}^{N, 1 / 2}$ fixed by $-\sigma_{*}$. This is isomorphic to $\mathcal{D}_{-, m}^{N}$.

Following the notation in Ref. $3, \mathcal{D}_{m}^{N, 1 / 2}$ gives rise to a $1 / 2 \mathrm{Z}$-twisted and $\mathbb{Z}_{2}$-local formal distributions algebra with the family

$$
\mathcal{F}=\left\{J_{A_{i}}^{n, i / 2, \pm 1}(z):=J^{n, i / 2, \pm 1}(z) \otimes A_{i}:=\sum_{k \in Z}( \pm t)^{k+i / 2}\left(-\partial_{t}\right)^{n} z^{-k-1-i / 2} A_{i}: A_{i} \in\left(\mathrm{Mat}_{N} \mathrm{C}\right)_{i}, i \in \mathbb{Z}_{2}\right\} .
$$

Observe that $J_{A_{i}}^{n, i / 2, \pm 1}(z)=\delta_{i / 2}(z-( \pm 1) t)(-\partial)^{n} A_{i}$ where

$$
\delta_{i / 2}(z-( \pm 1) t)=\sum_{k \in Z}(( \pm 1) t)^{k+i / 2} z^{-k-1-i / 2} .
$$

Its OPE is $\left(\alpha, \beta \in \mathbb{Z}_{2}\right)$ :

$$
\begin{aligned}
{\left[J_{A_{i}}^{m, i / 2, \alpha}(z), J_{A_{j}}^{n, i / 2, \beta}(w)\right]=} & -\sum_{s=0}^{n}\left(\begin{array}{l}
n \\
s
\end{array}\right)(-\beta)^{s} J_{A_{j} A_{i}}^{m+n-s,(i+j) / 2, \beta}(w) \partial_{w}^{s} \delta_{i / 2}\left(z-\alpha \beta^{-1} w\right) \\
& +\sum_{r=0}^{m} \sum_{k=0}^{r}\left(\begin{array}{c}
m \\
r
\end{array}\right)\left(\begin{array}{l}
r \\
k
\end{array}\right) \beta^{r}\left(\partial_{w}^{k} J_{A_{i} A_{j}}^{m+n-r,(i+j) / 2, \beta}(w)\right) \partial_{w}^{r-k} \delta_{i / 2}\left(z-\alpha \beta^{-1} w\right) .
\end{aligned}
$$

Now we also introduce the following operator on the space of $1 / 2 Z$-twisted and $Z_{2}$-local formal distributions: $T_{\alpha}(a(z))=\alpha a(\alpha z)$, with $\alpha \in \mathbb{Z}_{2}$. Note that $\mathcal{F}_{m}$ is closed under all $T_{\alpha}$.

Thus, introducing $\operatorname{symb}\left(J_{A_{i}}^{1, i / 2,1}\right)=x A_{i}$ and $\operatorname{symb}\left(J_{A_{i}}^{1, i / 2,-1}\right)=y A_{i}$ with $A_{i} \in\left(\mathrm{Mat}_{N} \mathrm{C}\right)_{i}, \quad i \in \mathbb{Z}_{2}$ $=\{0,1\}$, we have that the associated $\mathbb{Z}_{2}$-graded conformal algebra

$$
g c_{N, m}:=\overline{\mathcal{F}_{m}}=\underset{i \in \mathbb{Z}_{2}}{\bigoplus}\left(\left(\operatorname{Mat}_{N} \mathrm{C}[\partial, x]\right)_{i} \oplus\left(\operatorname{Mat}_{N} \mathrm{C}[\partial, y]\right)_{i}\right)
$$

with $\lambda$-bracket

$$
\begin{aligned}
& {\left[A_{i}(\partial, x)_{\lambda} B_{j}(\partial, x)\right]=A_{i}(-\lambda, \lambda+\partial+x) B_{j}(\lambda+\partial, x)-B_{j}(\lambda+\partial,-\lambda+x) A_{i}(-\lambda, x),} \\
& {\left[A_{i}(\partial, y)_{\lambda} B_{j}(\partial, y)\right]=A_{i}(-\lambda,-\lambda-\partial+y) B_{j}(\lambda+\partial, y)-B_{j}(\lambda+\partial, \lambda+y) A_{i}(-\lambda, y),}
\end{aligned}
$$

and $\left[A_{i}(\partial, x)_{\lambda} B_{j}(\partial, y)\right]=0$. Again, the $\mathbb{Z}_{2}$-action on it by semilinear automorphism is given by $T_{-1}\left(A_{i}(\partial, x)\right)=A_{i}(\partial, y)$ and $T_{-1}\left(A_{i}(\partial, y)\right)=A_{i}(\partial, x)$. 
Therefore, $g c_{N, m} \simeq g c_{N} \oplus g c_{N} \simeq \operatorname{Mat}_{N} \mathrm{C}[\partial, x] \oplus \operatorname{Mat}_{N} \mathrm{C}[\partial, y]$ endowed with a $\mathbb{Z}_{2}$-gradation and a $Z_{2}$-action by semilinear automorphisms given by $T_{-1}$.

It is easy to see that (in terms of symbol) we have for $i \in Z_{2}$,

$$
\begin{aligned}
& \sigma_{*}\left(A_{i}(\partial, x)\right)=A_{i}^{\ddagger}(\partial, y-\partial), \\
& \sigma_{*}\left(A_{i}(\partial, y)\right)=A_{i}^{\ddagger}(\partial, x+\partial) .
\end{aligned}
$$

Thus, we consider the $-\sigma_{*}$-fixed subalgebra which is the C-linear span of $\overline{A_{i}(\partial, x)}:=A_{i}(\partial, x)$ $-A_{i}^{\ddagger}(\partial, y-\partial)$.

The $\lambda$-bracket is $\left(i, j \in \mathbb{Z}_{2}\right)$

$$
\left[\overline{A_{i}(\partial, x)} \bar{B}_{B_{j}(\partial, x)}\right]=\overline{A_{i}(-\lambda, x+\lambda+\partial) B_{j}(\lambda+\partial, x)}-\overline{B_{j}(\lambda+\partial,-\lambda+x) A_{i}(-\lambda, x)} .
$$

Therefore, the conformal subalgebra associated with $\mathcal{D}_{-, m}^{N}$ is isomorphic to $g c_{N}$ with a $\mathbb{Z}_{2} \times \mathbb{Z}_{2}$-action given by a $\mathbb{Z}_{2}$-gradation (that comes from the $\mathbb{Z}_{2}$ gradation in $\mathrm{Mat}_{N} \mathrm{C}$ ) and $\mathbb{Z}_{2}$-action by semilinear automorphism given by $T_{1}=i d$ and $T_{-1}$.

\section{ACKNOWLEDGMENTS}

Part of this work was done during our visit to MIT in April 2000, we are very grateful for the hospitality at MIT. This research was supported in part by Foncyt, CONICET, Fomec, Conicor and SECYT (Argentina).

\section{APPENDIX}

Here we will prove

Theorem 2: The Lie algebras $\mathcal{D}_{ \pm, m}^{N}$ are simple.

Proof: We will give the proof only for the case $\mathcal{D}_{+, m}^{N}$, since the other is completely analogous. We are going to use the description in terms of generators given in Sec. IV. Since the proof is rather technical, some details will only be sketched. Assume that $b=1$. Let us define

$$
X=\left(\begin{array}{cc}
\left(D-\frac{1}{2}\right) I_{m} & 0 \\
0 & D I_{N-m}
\end{array}\right) \in \mathcal{D}_{+, m}^{N} .
$$

Note that $(k \in \mathbb{Z})$

$$
\operatorname{ad}(X)\left(t^{k} f\left(D+\frac{k-1}{2}\right) A=k t^{k} f\left(D+\frac{k-1}{2}\right) A,\right.
$$

where $A=\left(\begin{array}{cc}A_{1} & 0 \\ 0 & 0\end{array}\right)$. Similarly, if $F=\left(\begin{array}{ll}0 & 0 \\ 0 & F_{1}\end{array}\right)$, we have that

$$
\operatorname{ad}(X)\left(t^{k} f\left(D+\frac{k}{2}\right) F\right)=k t^{k} f\left(D+\frac{k}{2}\right) F
$$

and if $B C=\left(\begin{array}{cc}0 & B \\ t^{-1} C & 0\end{array}\right)$, we get

$$
\operatorname{ad}(X)\left(t^{k} f\left(D+\frac{k-1}{2}\right) B C\right)=\left(k-\frac{1}{2}\right) t^{k} f\left(D+\frac{k-1}{2}\right) B C .
$$


Let $\mathcal{J}$ be a nonzero ideal of $\mathcal{D}_{+, m}^{N}$ and let $v \in \mathcal{J}$ and $v=v^{A}+v^{B C}+v^{F}$, with $v^{A}+v^{F}$ of the form $\left(\begin{array}{cc}* & 0 \\ 0 & *\end{array}\right)$, and $v^{B C}$ of the form $\left(\begin{array}{ll}0 & * \\ * & 0\end{array}\right)$.

By $[(\mathrm{A} 2 \mathrm{a})-(\mathrm{A} 2 \mathrm{c})]$ we may assume that $v^{A}+v^{F} \in \mathcal{J}$ and $v^{B C} \in \mathcal{J}$. So, since $\left[I_{m}, v^{A}+v^{F}\right]$ $\in \mathcal{J}$ we have that $v^{A} \in \mathcal{J}$ and similarly we can show that $v^{F} \in \mathcal{J}$.

We are going to show that if $v^{A} \in \mathcal{J}, v^{A} \neq 0$, then $E_{i, m+1-j} \pm E_{j, m+1-i} \in \mathcal{J}$ and $E_{i, m+1-i} \in \mathcal{J}$ with $1 \leqslant i, j \leqslant m$. Analogous arguments will work finely for the remaining blocks, and the details are left to the reader. Therefore we can conclude that $\mathcal{J}=\mathcal{D}_{+, m}^{N}$.

Take $v^{A} \neq 0$ in $\mathcal{J}$. By (A2a), we may suppose $v^{A}=t^{k} A(D)=t^{k} \sum_{1 \leqslant p, j \leqslant m} a_{p j}(D) E_{p, j}$ with $A$ of the form $\left(\begin{array}{cc}* & 0 \\ 0 & 0\end{array}\right)$.

Assume that $a_{i, s}(D) \neq 0$. Thus,

$$
\begin{aligned}
{\left[\left(D-\frac{1}{2}\right) E_{i, m+1-i,} t^{k} A(D)\right]=} & t^{k} \sum_{1 \leqslant j \leqslant m}\left\{\left(D+k-\frac{1}{2}\right) a_{m+1-i, j}(D) E_{i, j}\right. \\
& \left.-\left(D-\frac{1}{2}\right) a_{j, i}(D) E_{j, m+1-i}\right\} .
\end{aligned}
$$

Now take $Y=t^{-k}\left(E_{r, s}-E_{m+1-s, m+1-r}\right) \in \mathcal{D}_{+, m}^{N}$ with $s \neq m+1-r, s \neq i$ and $r \neq m+1-i$. Computing $\operatorname{ad}(Y)$ of (A3) we get

$$
\begin{gathered}
\left(D-\frac{1}{2}\right)\left[a_{m+1-i, m+1-s}(D-k) E_{i, m+1-r}-a_{i, s}(D) E_{r, m+1-i}\right. \\
\left.+a_{m+1-r, i}(D) E_{m+1-s, m+1-i}-a_{m+1-i, r}(D-k) E_{i, s}\right]
\end{gathered}
$$

and again, $\operatorname{ad}\left((D-1 / 2) E_{m+1-r, r}\right)(r \neq m+1-r, r \neq i)$, applied to (A4), gives us

$$
-\left(D-\frac{1}{2}\right)^{2}\left[a_{m+1-i, m+1-s}(D-k) E_{i, r}+a_{s, i}(D) E_{m+1-r, m+1-i}\right] \in \mathcal{J}
$$

with $a_{s, i}(D) \neq 0$. In particular, (A5) belongs to $\mathcal{D}_{+, m}^{N}$, thus we have that

$$
a_{m+1-i, m+1-s}(D)=-a_{s, i}(-D-k+1) .
$$

Therefore, we may assume that $-(D-1 / 2)^{2}\left[f(D) E_{i, r}+f(-D-k+1) E_{m+1-r, m+1-i}\right] \in \mathcal{J}$ for some $f \neq 0$.

Note that $E_{p q}-E_{m+1-q, m+1-p} \in \mathcal{D}_{+, m}^{N}$ for any $1 \leqslant p, q \leqslant m$. If we pick different values for $p$ and $q$ it is easy to show that

$$
-\left(D-\frac{1}{2}\right)^{2}\left[f(D) E_{p, q}+f(-D-k+1) E_{m+1-q, m+1-p}\right] \in \mathcal{J},
$$

for arbitrary values of $p$ and $q$. Note in particular, if $p=m+1-q$ we get an element on the diagonal (cf. with description of generators in Sec. IV).

Now to finish the proof, we only need to show we can lower the degree of $f(D)$ in (A7). But again, since $t\left(E_{l l}-E_{m+1-l, m+1-l}\right) \in \mathcal{D}_{+, m}^{N}$ for $1 \leqslant l \leqslant m$, and if we compute the bracket between (A7) and $t\left(E_{p, p}-E_{m+1-p, m+1-p}\right)$, and the bracket between (A7) and $t\left(E_{q, q}-E_{m+1-q, m+1-q}\right)$ and then the difference between them we have that

$$
t\left((f(D+1)-f(D)) E_{p, q}+(g(D)-g(D-1)) E_{m+1-q, m+1-p}\right) \in \mathcal{J} .
$$

Thus we get an element in the ideal with lower degree in $D$, but we have increased the degree in $t$. Using the argument in (A4), we can again lower the degree in $t$. This process will eventually end, showing that $E_{p, q} \pm E_{m+1-q, m+1-p}$.

${ }^{1}$ V. Kac, W. Wang, and C. Yan, "Quasifinite representations of classical Lie subalgebras of $W_{1+\infty}$," Adv. Math. 139, 56-140 (1998)

${ }^{2}$ V. Kac, Vertex Algebras for Beginners, 2nd ed. American Mathematical Society, Providence, Rhode Island (1998).

${ }^{3} \mathrm{~V}$. Kac, "Formal distributions algebras and conformal algebras," in Proceedings of the XIIth International Congress of 
Mathematical Physics (ICMP '97) (Brisbane) (International, Cambridge, 1999), pp. 80-97, preprint math.QA/9709027.

${ }^{4}$ M. Golenishcheva-Kutuzova and V. Kac, "T-conformal algebras," J. Math. Phys. 39, 2290-2305 (1998).

${ }^{5}$ C. Boyallian, V. Kac, J. Liberati, and C. Yan, "Quasifinite highest weight modules over the Lie algebra of matrix differential operators on the circle," J. Math. Phys. 39, 2910-2928 (1998). 